\title{
Leadership des filles et mentoring
}

Karen Austrian

Population Council

Follow this and additional works at: https://knowledgecommons.popcouncil.org/departments_sbsr-pgy

Part of the Family, Life Course, and Society Commons, Gender and Sexuality Commons, Gender Equity in Education Commons, and the International Public Health Commons How does access to this work benefit you? Let us know!

\section{Recommended Citation}

Austrian, Karen. 2012. "Leadership des filles et mentoring," GIRLS FIRST! Perspectives on Girl-Centered Programming. New York: Population Council. 
UNE PUBLICATION GIRLS FIRST!

LEADERSHIP

DES FILLES

ET

MENTORING

\section{PAR KAREN AUSTRIAN}

BILAN

POLITIQUE/

PROGRAMMATION

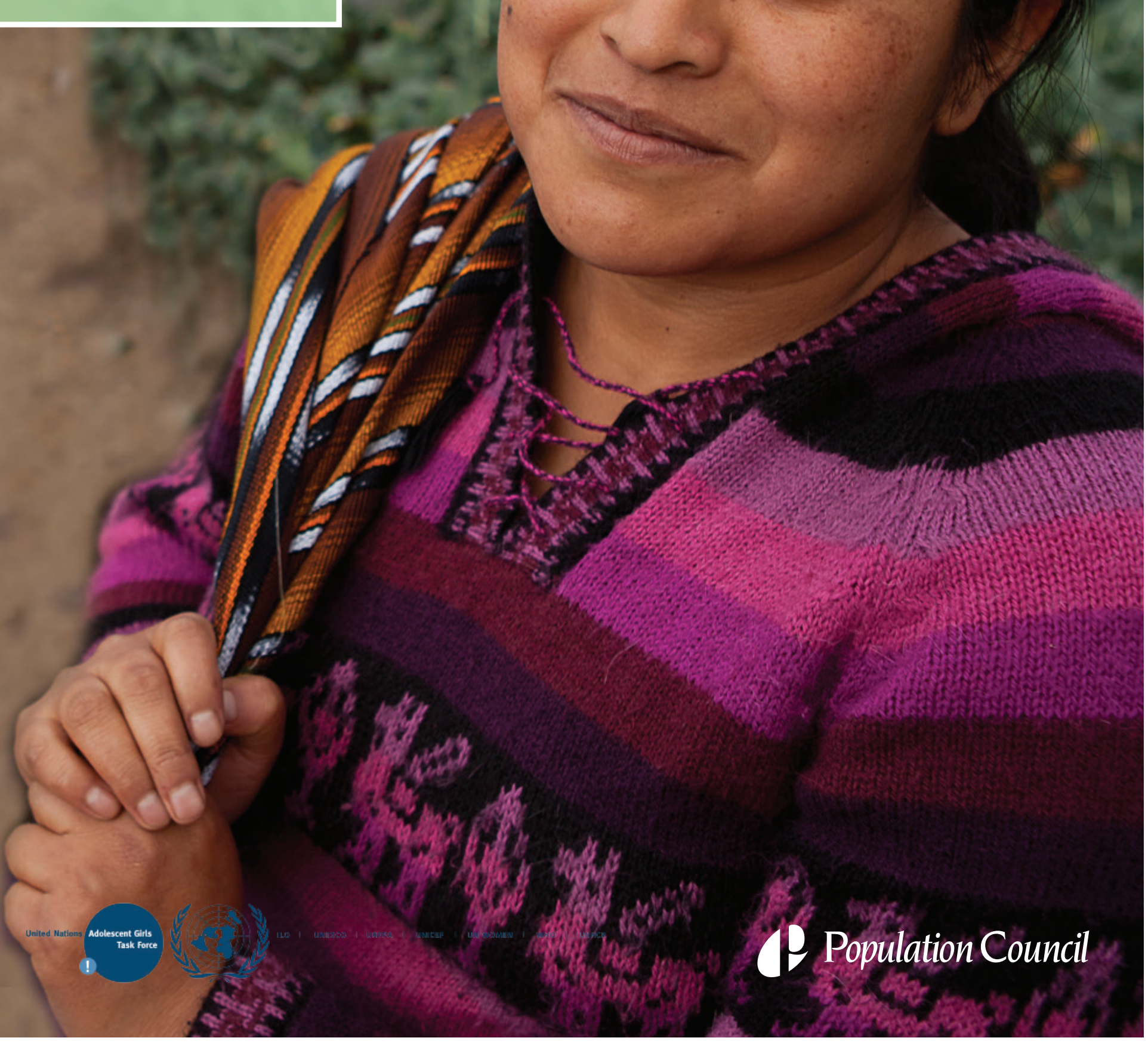




\section{GIRLS FIRST!}

\section{PERSPECTIVES DE PROGRAMMATION CENTRÉE SUR LES FILLES}

La recherche et les programmes consacrés aux adolescentes se concentrent traditionnellement sur la sexualité, la santé génésique et le comportement, négligeant la problématique socioéconomique plus large qui sous-tend leurs droits humains, leur développement général, leur santé et leur bienêtre. De plus, les efforts d'amélioration de la vie des filles mettent souvent en vedette ceux et celles qui dominent ou influencent leur vie (les parents, la belle-famille, les garçons, les hommes, les agresseurs), sans prêter aux intéressées elles-mêmes l'attention qu'elles méritent en soi.

GIRLS FIRST! Perspectives de programmation centrée sur les filles est une série de cinq comptes rendus thématiques, rédigés par les experts et expertes du Population Council. Ces dossiers présentent un instantané de la base de connaissances telle qu'elle se présente, à un moment donné, dans ce domaine en évolution permanente. Ils abordent les cinq priorités stratégiques définies dans la Déclaration conjointe des Nations Unies, "Intensifier l'action menée pour promouvoir les droits des adolescentes " (mars 2010), symbole de l'engagement collectif de sept organismes de l'ONU au soutien des gouvernements et de leurs partenaires pour la promotion de politiques et programmes essentiels au profit des adolescentes les plus difficiles à atteindre. Ces comptes rendus

1. explorent l'évolution prochaine de l'éducation des filles ;

2. définissent les grandes lignes d'approches innovantes en vue de l'amélioration de la santé des filles;

3. recadrent l'approche de la violence à l'encontre des filles;

4. décrivent les meilleurs moyens de cultiver les leaders féminines; et

5. expliquent de nouveaux modes inédits de collecte et d'exploitation des données relatives aux adolescentes.

Ils avancent des arguments novateurs en faveur de l'investissement dans les filles et mettent en valeur les pratiques prometteuses. Ils expriment un point de vue factuel progressiste quant aux allocations de ressources aptes à assurer le plus rapidement et le plus efficacement l'amélioration de la vie des filles.

Rédigés par des experts et expertes du Population Council, une organisation pionnière d'une recherche et programmation de pointe à l'intention des adolescentes vulnérables et marginalisées, ces rapports ont bénéficié, d'abord et surtout, de l'appui et du leadership de l'Équipe spéciale des Nations Unies pour les adolescentes. L'initiative a également reçu l'appui moral et financier de la Nike Foundation, de la Fondation des Nations Unies et de la David and Lucile Packard Foundation. Ces comptes rendus viennent s'ajouter à la guidance programmatique et aux " boîtes à outils " aujourd'hui disponibles ; ils offrent une ressource de référence essentielle à toute initiative d'élaboration de politiques et programmes efficaces et durables au profit des filles. Notre espoir est qu'ils inspireront de nouvelles approches utiles aux efforts de réalisation des droits des adolescentes marginalisées du monde.

Août 2012

Population Council et Équipe spéciale des Nations Unies pour les adolescentes

Avis

Ces comptes rendus sur la programmation pour adolescentes dans les domaines de l'éducation, de la santé, de la réduction de la violence, du leadership des filles et des données ont été préparés par le Population Council pour l'Équipe spéciale des Nations Unies pour les adolescentes, avec l'appui de la Fondation des Nations Unies, de la Nike Foundation et de la Packard Foundation.

Les opinions exprimées sont strictement celles des auteurs et ne reflètent pas nécessairement celles des partenaires donateurs. 


\title{
LEADERSHIP DES FILLES ET MENTORING
}

\author{
PAR KAREN AUSTRIAN
}

\section{CONNAISSANCES ET PRINCIPES FONDAMENTAUX}

Par rapport aux garçons, les filles ont moins l'occasion de rencontrer leurs amies, de se faire de nouveaux amis et de bénéficier d'échanges avec leurs mentors

Il y a dans toutes les communautés du monde des filles dotées d'un potentiel de leadership 2

Les cadettes ont besoin de leaders locales qu'elles puissent admirer et imiter

La possibilité d'être vue comme leader et mentor au sein d'une communauté

transforme la perception que la fille a d'elle-même

La présence d'un réseau de jeunes leaders féminines (et d'un espace sûr qu'elles occupent régulièrement) remet implicitement et explicitement en question la structure

du pouvoir au sein de la communauté et donne une forte voix féminine aux structures décisionnelles

\section{POLITIQUES ET PRATIQUES PRÉCONISÉES}

Leadership en cascade : Partir du leadership féminin existant et construire les modèles programmatiques sur cette base

Former des adolescentes plus âgées ou des jeunes femmes à devenir des leaders au sein de leurs propres communautés, responsables des programmes proposés à leurs cadettes

Soutenir les mentors en leur offrant une rémunération appropriée

Assurer l'encadrement et l'accompagnement continus des mentors

Faire participer les filles à l'identification du contenu et des méthodes les mieux adaptés

Employer des leaders et des mentors qui sont elles-mêmes des filles; elles sont souvent les plus efficaces et les mieux placées à long terme pour apporter le contenu visé aux autres filles de leur communauté

Reconnaître que mentors et pairs éducatrices constituent deux modèles différents au service d'objectifs distincts

Faire l'expérience du contenu une fois la structure en place

RESSOURCES : PROGRAMMES “ LABORATOIRES D’APPRENTISSAGE »

Programme des espaces sûrs de Binti Pamoja (Filles unies) : Renforcement des compétences des adolescentes de 10 à 19 ans de Kibera (Kenya)

Safe and Smart Savings Products for Vulnerable Adolescent Girls in Kenya and Uganda :

Renforcer le leadership des filles et leur accès aux produits financiers

Abriendo Oportunidades (Opening Opportunities): Programme destiné aux filles indigènes de

8 à 18 ans des communautés rurales les plus pauvres du Guatemala

Biruh Tesfa (Avenir prometteur): Programme destiné aux employées de maison et migrantes ruro-urbaines dans les bas-quartiers d'Éthiopie

Conception d'un programme centré sur les filles : Boîte à outils pour l'élaboration, le renforcement et l'expansion de programmes destinés aux adolescentes 
CONNAISSANCES

ET PRINCIPES FONDAMENTAUX

Par rapport aux garçons, les filles ont moins l'occasion de rencontrer leurs amies, de se faire de nouveaux amis et de bénéficier d'échanges avec leurs mentors ${ }^{1}$

Plusieurs enquêtes d'évaluation des réseaux sociaux des filles indiquent que ces dernières ne bénéficient pas des mêmes débouchés sociaux que les garçons. Par exemple, dans le bidonville de Kibera à Nairobi (Kenya), une enquête auprès des adolescents a révélé que si $70 \%$ des garçons avaient de nombreux amis dans leur quartier, il n'en allait ainsi que pour $50 \%$ des filles. En outre, alors que plus de deux tiers des garçons disposaient d'un endroit en dehors de leur propre foyer, de celui de leurs amis ou de l'école où rencontrer leurs amis de même sexe, un tiers seulement des filles avaient accès à un tel endroit ${ }^{2}$. Dans les bas quartiers d'Addis Abeba (Éthiopie), 60\% des garçons ont "beaucoup d'amis ", par rapport à $24 \%$ des filles seulement. Cinquante pour cent des garçons ont un endroit où rencontrer leurs amis de même sexe, par rapport à $13 \%$ des filles seulement ${ }^{3}$. Dans la région rurale d'Amhara (Éthiopie), 68\% des garçons, par rapport à $59 \%$ des filles, disent avoir " beaucoup d'amis ". Près de la moitié des garçons ont un endroit où rencontrer leurs amis de même sexe, par rapport à $16 \%$ des filles ${ }^{4}$.

Le renforcement de l'actif social critique (tel qu'amies et mentors) amoindrit les risques que courent les filles sur le plan de la santé et de leur situation économique.

Les programmes de qualité organisés à l'intention des filles et des jeunes femmes dans les communautés défavorisées renforcent leurs capacités de leadership et leur ouvrent de nouveaux horizons. Les filles et les jeunes femmes des communautés bénéficiaires de ces programmes doivent être soutenues et accompagnées jusqu'à ce qu'elles maîtrisent les compétences adéquates pour assumer elles-mêmes le leadership et le mentoring des autres filles de la communauté.
Il y a dans toutes les communautés du monde des filles dotées d'un potentiel de leadership.

Ces filles constituent une ressource extrêmement précieuse pour les communautés les plus démunies car ce potentiel peut en faire les leaders et mentors des autres filles de la communauté. L'avantage du renforcement des capacités de leadership de ces filles est triple : il définit les héroïnes et les modèles à suivre des jeunes filles, il autonomise les aînées et les rend plus fortes, et il remet en question les normes dépassées de la communauté.

Les cadettes ont besoin de leaders locales qu'elles puissent admirer et imiter.

Quand une fille se trouve en situation de crise et a besoin de quelqu'un vers qui se tourner, son mentor doit être dans la communauté, prête et apte à répondre. Trop souvent, les gestionnaires de programme estiment que les leaders ou les mentors doivent être des étudiantes universitaires ou de jeunes femmes professionnelles importées de communautés mieux loties. Ces jeunes femmes ont beau être motivées et bienveillantes, elles n'ancrent pas le leadership au sein de la communauté défavorisée. Par exemple, la scolarisation des jeunes Africaines de 19 ans est la plus faible dans le sud du continent. Plus de $40 \%$ de ces filles ont achevé l'école au Ghana, par rapport à environ $2 \%$ seulement au Mozambique. Sur la plupart du continent, au moins cinq pour cent des filles sont déscolarisées avant l'âge de 19 ans $^{5}$. Les filles qui ont achevé l'école ont un potentiel de leadership et peuvent servir de mentors à leurs cadettes au sein de la communauté. Ces filles ne sont d'ailleurs pas les seules à pouvoir assumer le rôle de mentors. Le talent et la volonté existent dans toutes les communautés, même les plus démunies. Ce sont les occasions qui, plus souvent, doivent être créées. II est impératif d'investir dans le renforcement du leadership de filles indigènes locales et d'assurer l'établissement d'un cadre de jeunes leaders féminines au sein de la communauté.
La possibilité d'être vue comme leader et mentor au sein d'une communauté transforme la perception que la fille a d'elle-même.

Avoir un rôle visible au sein de la communauté et un sentiment de responsabilité vis-à-vis de ses cadettes constitue une force de changement positive dans la vie des jeunes leaders féminines ${ }^{6}$. Voici ce qu'en disent deux leaders d'un programme pour filles organisé dans un bidonville du Kenya :

"Les filles comptent sur moi ; je dois montrer le bon exemple. Je suis fière du courage et du moral que j'ai pour les accompagner. Je suis fière de leur bonne conduite et du fait qu'elles sont conscientes de leurs droits. "

"Ce rôle me plaît. Les filles viennent me demander conseil. On se sent importante ; qu'on est quelqu'un dans la communauté."

La présence d'un réseau de jeunes leaders féminines (et d'un espace sûr qu'elles occupent régulièrement) remet implicitement et explicitement en question la structure du pouvoir au sein de la communauté et donne une forte voix féminine aux structures décisionnelles.

La plupart des communautés pauvres sont aussi dénuées d'un solide leadership féminin public - de femmes jeunes ou plus âgées -, ainsi que de lieux de rencontre féminins. Hallman a constaté que les réseaux d'amitié des filles sont beaucoup moins développés que ceux ces garçons (généralement, en Afrique du Sud, les garçons les plus pauvres ont plus d'amis et plus de contacts réguliers avec eux que les filles les plus riches) et, observation cruciale, qu'un plus grand isolement social présente une forte corrélation avec l'expérience de rapports sexuels forcés 7,8 .

L'ouverture de créneaux de leadership aux filles et aux jeunes femmes peut transformer leur position dans la collectivité et les habiliter à revendiquer leurs droits ${ }^{9}$. Par exemple, le Guatemala a adopté en 1996 une loi stipulant que toutes les communautés doivent prévoir un endroit où les filles et les femmes puissent se rencontrer. Dès le moment où un cadre de leaders féminines se développe, elles peuvent 
commencer à revendiquer ces espaces. La participation à un tel réseau de leadership accroît le respect dont bénéficient les filles et les femmes auprès des autres membres de la communauté. II n'est pas rare de voir les filles en position de leadership déclarer qu'elles ne subissent plus le harcèlement des hommes en raison du statut dont elles jouissent désormais dans la communauté. Placer les jeunes femmes dans ces positions de leadership est l'une des filières de transformation des normes de genre et de remise en question des structures et de la dynamique traditionnelles du pouvoir.

\section{POLITIQUES ET PRATIQUES PRÉCONISÉES}

\section{Leadership en cascade : Partir du leadership féminin existant et construire les modèles de premières mentors. Les éléments critiques du modèle de leadership en cascade sont les suivants :} programmatiques sur cette base. Le "leadership en cascade " offre un modèle de développement du leadership des filles couronné de succès dans différents contextes - des bidonvilles urbains d'Afrique orientale aux hauts-plateaux mayas du Guatemala et à la société conservatrice de la Haute Égypte. Dans ce modèle, les adolescentes plus âgées et les jeunes femmes, généralement de 18 à 30 ans, sont formées au mentoring de leurs cadettes au sein de la communauté. L'approche peut se réaliser sur une période prolongée, tandis que les filles suivent et achèvent elles-mêmes les activités du programme et apprennent à assumer des positions de leadership de plus en plus élevées, prenant notamment la tête des groupes de nouvelles inscrites. Le plus souvent, au niveau de la première génération, le programme ne dispose cependant pas d'un temps initial suffisant pour permettre aux filles de suivre un cycle complet de développement de leadership et il est nécessaire de former spécifiquement un groupe d'adolescentes plus âgées au rôle

Former des adolescentes plus âgées ou des jeunes femmes à devenir des leaders au sein de leurs propres communautés, responsables des programmes proposés à leurs cadettes. Les mentors sont généralement de jeunes femmes (généralement âgées de 18 à 30 ans) issues de la communauté, avec lesquelles les filles peuvent s'identifier. En plus de l'exécution du programme, les mentors servent d'exemples à suivre pour les filles. Contrairement aux programmes animés par les pairs, établis pour les populations scolarisées mieux loties, les mentors sont légèrement plus âgées que les filles inscrites au programme. Même dans la détresse, on trouve fréquemment des filles scolarisées un peu plus que les autres et qui possèdent les aptitudes nécessaires pour devenir mentors.

\section{Soutenir les mentors en leur offrant une rémunération appropriée.}

II ne faut surtout pas négliger le fait que les mentors et leaders de groupe doivent être rémunérées et traitées comme si elles occupaient un emploi (car elles travaillent effectivement !) Idéalement, il conviendrait de leur verser un petit salaire représentatif des heures qu'elles travaillent, calculé suivant celui payé pour d'autres emplois comparables dans la communauté (gardienne d'enfants, agent sanitaire de proximité, institutrice primaire, etc. ${ }^{10}$ Si cela n'est pas possible, les

\section{MODĖLE DE LEADERSHIP EN CASCADE}

Soutien national/régional

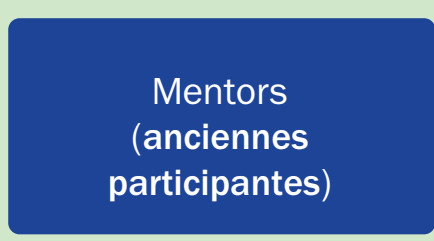

\section{Intervention de niveau communautaire}

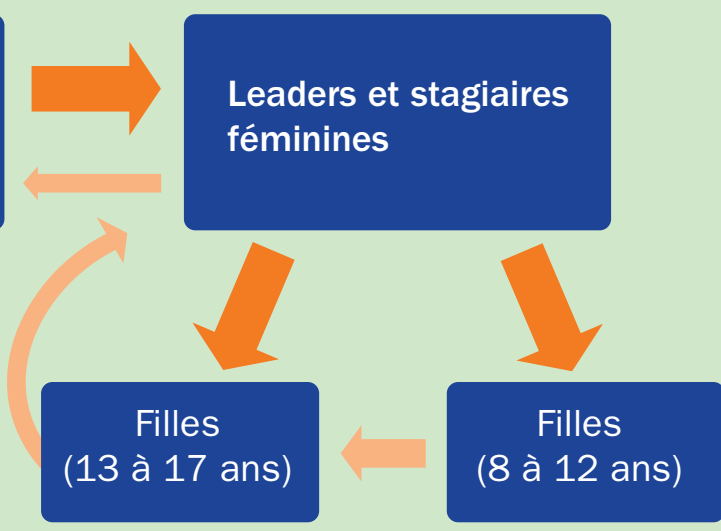


mentors et leaders de groupe peuvent être rémunérées par des formations, d'autres débouchés ou formes de compensation convenues avec elles. L'expérience révèle cependant qu'une petite compensation monétaire est le meilleur moyen d'assurer travail de qualité, engagement et durabilité. Les mentors payées sont plus susceptibles d'être présentes, de bien se préparer aux rencontres du groupe et d'assurer un soutien permanent aux filles de la communauté.

Assurer l'encadrement et l'accompagnement continus des mentors.

Cet encadrement peut prendre la forme de réunions mensuelles, d'entretiens personnels ou de visites de site, suivant l'étendue géographique du programme. Nous recommandons une forme de rencontre qui permette aux mentors de dialoguer, de partager leurs succès et leurs difficultés - concernant les pressions de mariage, d'inceste ou de violence que leur divulguent les filles, par exemple -, d'échanger leurs idées et les leçons tirées de leur expérience, et de construire leurs propres réseaux sociaux.

Faire participer les filles à l'identification du contenu et des méthodes les mieux adaptés.

Les filles leaders, en particulier celles qui sont capables de s'exprimer et qui posent un regard critique sur leur entourage, ont les meilleures perspectives des problèmes, difficultés et obstacles rencontrés par les autres filles de la communauté. Elles comprennent bien aussi les sujets importants à couvrir. II ne faudrait pas omettre leur participation au contenu du programme, à l'identification des thèmes principaux à aborder et aux préoccupations de sécurité des filles, notamment. II est utile de leur demander où et quand les filles peuvent se réunir, quels adultes sont importants dans leur vie, les événements saisonniers qui présentent le plus de risques pour elles (comme les vacances scolaires ou les festivals de la communauté), et comment le programme pourrait être adapté pour répondre à leurs plus grandes préoccupations ${ }^{11}$. Le Population Council a mis au point un outil d'évaluation de la sécurité destiné aux filles et aux garçons, pour les aider à définir les moments de la journée, de la semaine ou de l'année, ou bien les situations qui représentent pour eux une menace potentielle. L'outil d'évaluation de la sécurité jette les bases d'une discussion pratique de ce à quoi pourrait ressembler un plan de sécurité12,13.

Employer des leaders et des mentors qui sont elles-mêmes des filles; elles sont souvent les plus efficaces et les mieux placées à long terme pour apporter le contenu visé aux autres filles de leur communauté.

Le rassemblement régulier des filles, en sécurité, est la plus grande priorité. La matière couverte à l'occasion de ces rencontres est également importante. Au début surtout, elle doit être provisoire et évoluer suivant les besoins des participantes. Le contenu doit être adapté en fonction de leurs compétences et des exigences programmatiques. Les filles leaders et mentors doivent être à même de délivrer le contenu dans un langage et un style que les participantes comprennent et qui les mettent à l'aise. Leur maîtrise de la matière et leur aptitude à la transmettre aux autres représentent potentiellement une ressource communautaire à long terme. Le matériel de contenu et de formation utilisé dans un contexte doit souvent être adapté pour convenir à un autre. L'importance de ces ajustements ne doit pas être sous-estimée. L'adaptation du matériel au contexte local facilite la tâche de dissémination demandée des leaders, tout en le rendant plus pertinent à la vie des participantes. Par exemple, le contenu d'un programme de compétences financières peut être fixé, et même gradué en fonction de l'âge (modules élémentaires pour filles de 10 à 14 ans et de 15 à 19 ans). Les leaders chargées de la présentation peuvent disposer de 30 minutes d'instruction par session. Le jalonnement de la matière et son niveau doivent être évalués et adaptés soigneusement aux aptitudes des leaders et aussi à leurs conditions de travail. Un plan doit en outre être mise en place pour rafraîchir leur formation et assurer un certain niveau de contrôle de qualité de leur travail ${ }^{14}$.

Reconnaître que mentors et pairs éducatrices constituent deux modèles différents au service d'objectifs distincts.

Le modèle Mentors/Leadership en cascade implique le mentoring des cadettes par leur aînées. Même si toutes sont adolescentes ou jeunes femmes, il y a entre elles une différence d'âge : une fille de 24 ans dirige par exemple un groupe de filles de 12 à 14 ans, ou une fille de 15 ans en guide une de 10 ans. L'aspect critique de l'approche est qu'elle donne aux aînées un rôle de leadership et certaines responsabilités, et aux cadettes un exemple de ce qu'elles peuvent aspirer à devenir dans le futur (un but à atteindre).

En revanche, l'éducation par les pairs est une approche de transfert d'information entre jeunes de même âge. Ce modèle s'est avéré bénéfique aux pairs éducatrices elles-mêmes, mais moins aux participantes aux programmes ${ }^{15,16}$. II tend par ailleurs à élever les jeunes moins vulnérables aux positions de leadership : les filles sélectionnées le sont en effet souvent parce qu'elles se distinguent déjà au sein du groupe. Enfin, l'éducation par les pairs ne construit pas une infrastructure durable de leadership et de présentation et poursuite du programme par les filles, car les pairs éducatrices suivent elles-mêmes le cycle du programme, sans y rester attachées après sa conclusion.

Faire l'expérience du contenu une fois la structure en place.

Les filles leaders peuvent être considérées un peu comme des agents de vulgarisation de communauté. Leur présence constante dans la communauté leur permet de repérer les filles les plus aptes à bénéficier des programmes et à gagner leur confiance. Bien que non encore es- 
sayée ni évaluée à cet égard, il serait utile d'explorer l'utilité de cette structure de leadership dans le secteur de la santé, de la vie civique et des compétences financières. Sur le plan de la santé, il serait concevable que les filles puissent devenir sources d'information sanitaire, de produits, de services d'orientation et peut-être même de vaccins. Peut-être pourrait-on par exemple former les filles leaders à offrir le vaccin anti-HPV aux adolescentes de leur communauté. Sur le plan civique, on pourrait imaginer de les mobiliser pour assurer que toutes les filles de la communauté soient titulaires de leur documentation d'identité indispensable (extrait d'acte de naissance, carnet de vaccination, etc.) Les filles leaders pourraient éventuellement être reconnues comme "leaders locales " aptes à signer les lettres de vérification aux fins de participation au scrutin, d'ouverture de compte, etc. Et sur le plan financier, peut-être pourraient-elles jouer un rôle fondamental dans la prestation d'une certaine éducation et de services financiers.
Ce modèle programmatique s'est révélé efficace tant en termes d'enrichissement de l'expérience des filles - par l'élargissement de leurs réseaux sociaux et de leur sécurité et par l'offre d'une source de soutien et d'une vision pour l'avenir - qu'au niveau de l'ouverture de nouveaux débouchés de leadership positif aux adolescentes plus âgées et aux jeunes femmes elles-mêmes. Le leadership en cascade n'a pas encore été testé dans toutes les circonstances où il semble prometteur, mais le potentiel est là et l'expérience est encouragée.

\section{RESSOURCES :}

PROGRAMMES

" LABORATOIRES

D'APPRENTISSAGE "

Programme des espaces sûr de Binti Pamoja (Filles unies) : Renforcement des compétences des adolescentes de 10 à 19 ans de Kibera (Kenya)

Binti Pamoja est un programme destiné aux adolescentes de 10 à
19 ans du bidonville de Kibera à Nairobi (Kenya). Le programme s'est développé en 2002 autour d'un groupe initial de 15 filles, bientôt divisé en deux groupes. Les filles ont grandi avec le programme et, au bout de deux ans, il est devenu clair qu'elles étaient prêtes à " recevoir leur diplôme " et à assumer des rôles de leadership, alors même que le programme était prêt à s'étendre dans la communauté. En 2006, la promotion initiale du programme a donc suivi une formation appelée à renforcer les compétences des filles sur le plan de l'animation de rencontre, du développement de groupe, de la communication et de la résolution de conflit. Cela fait, elles ont été affectées, deux par deux, à la formation de leurs propres groupes de rencontre dans la communauté. Les mentors /anciennes élèves ont assumé la responsabilité du recrutement des filles, de la recherche d'espaces de rencontre au sein de la communauté, de la planification et de l'animation des rencontres hebdomadaires, du soutien et conseil

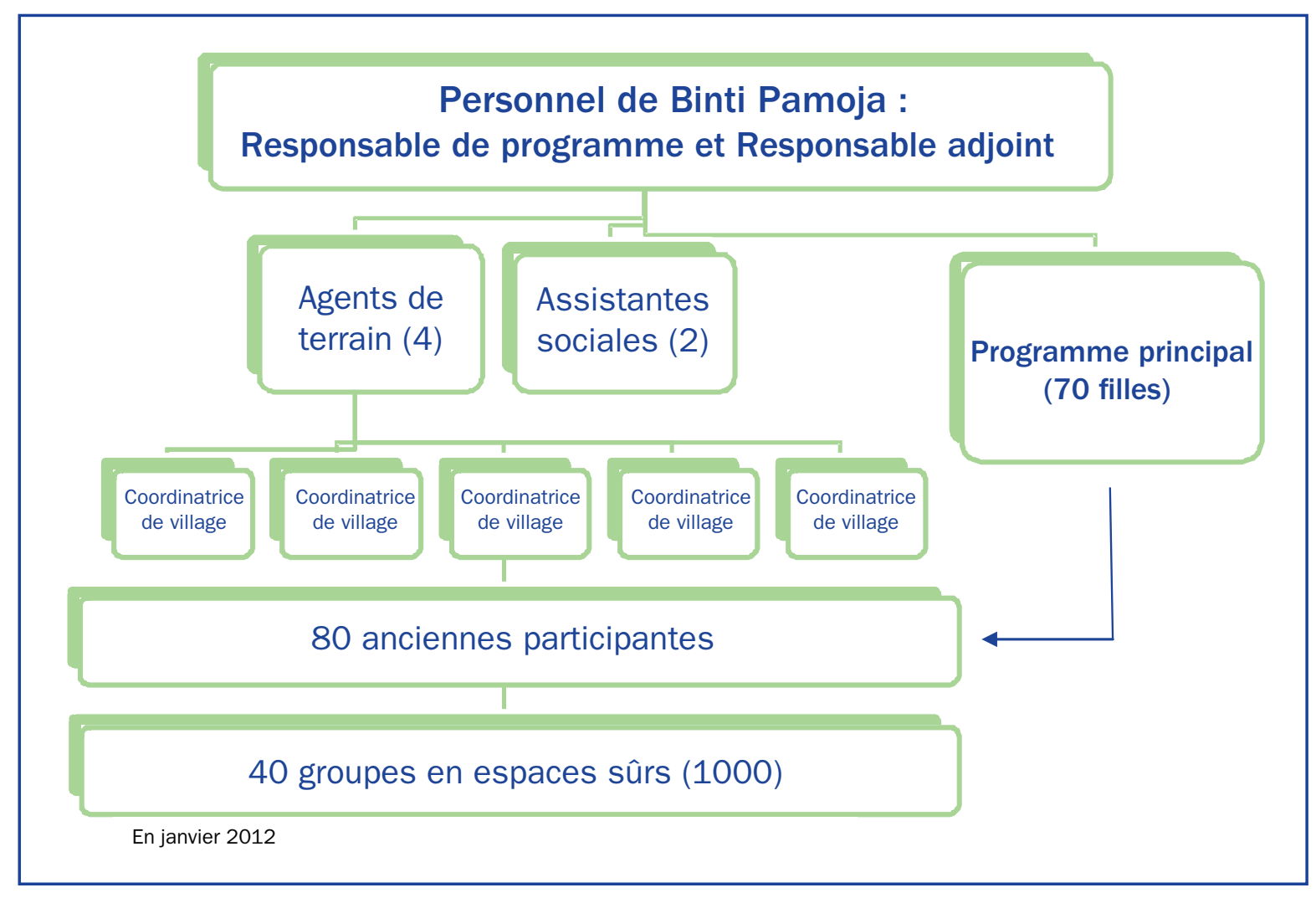


apportés aux filles et de la gestion du budget mensuel du groupe. Ces anciennes touchent un petit salaire et participent chaque mois à des réunions d'encadrement avec le personnel de Binti Pamoja.

En un an, le " programme des espaces sûrs " de Binti Pamoja comptait 20 anciennes élèves et 10 groupes. Devenu trop grand pour ses deux responsables originales, il a engagé deux autres anciennes au titre d'agents de terrain à temps partiel, les chargeant de l'encadrement de celles responsables des espaces sûrs et de leurs groupes respectifs. Le programme a continué à grandir, d'année en année, tandis que de nouvelles filles achevaient le cycle principal de Binti, devenaient à leur tour anciennes élèves et formaient leurs propres groupes dans la communauté. Dès 2009, il comptait 50 anciennes élèves et 25 groupes et devenait, une fois encore, trop vaste pour ses deux agents de terrain. Un nouvel échelon de leadership a donc été introduit entre les anciennes/ leaders de groupe et les agents de terrain : les coordinatrices de village. Au nombre de cinq, celles-ci sont responsables, chacune, d'un secteur géographique précis de Kibera. Elles supervisent chacune quatre à six groupes de filles. Elles aident les anciennes élèves à recruter, à identifier les espaces de rencontre appropriés, à superviser le contenu abordé par le groupe et à planifier les événements et les formations.

Suivant le modèle du leadership en cascade, Binti Pamoja a pu grandir, d'un programme initial de 40 filles se réunissant en un seul emplacement de Kibera à un programme de plus de 1000 filles, 40 groupes (de 20 à 30 filles chacun), 80 anciennes élèves assumant un rôle de leadership ou l'autre, avec une présence dans chaque village de Kibera.

Pour plus de détails, voir le site Web de du programme : http://cfk.unc.edu/whatwedo/ daughtersunited/ [en anglais].

\section{Safe and Smart Savings Products for Vulnerable Adolescent Girls in Kenya and Uganda : Renforcer le leadership des filles et leur accès aux produits financiers}

Ce programme, géré en partenariat par le Population Council et MicroSave Consulting, Ltd., en association avec les institutions financières FauluKenya et K-Rep Bank au Kenya et Finance Trust et FINCA-Uganda en Ouganda, cherche à ouvrir un compte d'épargne formel au nom des adolescentes participantes. Les comptes sont proposés selon le " modèle des espaces sûrs ": les filles sont organisées en groupes qui se rencontrent chaque semaine, pour apprendre et parler de questions élémentaires de santé et de finances. Chaque groupe a pour mentor une jeune femme de la communauté qui reçoit un petit salaire pour son travail, anime les réunions du groupe et aide à mobiliser les filles. Les mentors reçoivent une formation de formatrices intense en début de cycle, en matière de finances et de compétences d'animation et de gestion de groupe, et elles participent ensuite chaque mois à des réunions d'encadrement avec le personnel du programme.

Ce programme représente un exemple de leadership en cascade non organique, faute de temps durant la phase d'établissement (il aurait fallu attendre que les premières inscrites aux groupes d'épargne grandissent et mûrissent avant de devenir mentors). Les mentors ont été sélectionnées et formées à la fois au contenu délivré aux filles et aux compétences de leadership, de communication et d'animation de groupe.

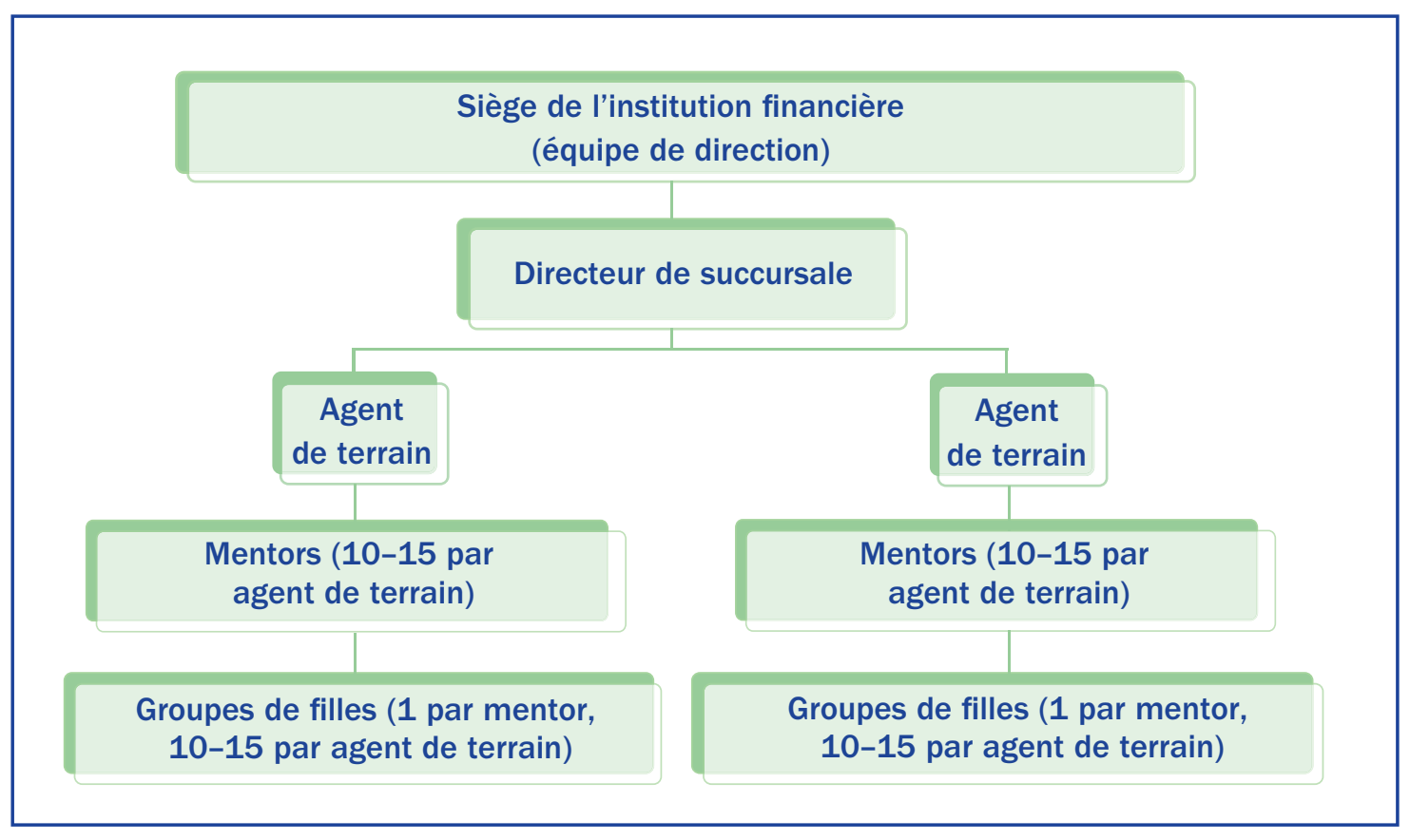


En ce qui concerne les établissements financiers, chaque branche offrant le produit est encadrée par un responsable de branche. Un ou deux agents de terrain sont spécifiquement responsables des comptes d'épargne des filles et de la liaison avec les mentors. Suivant l'établissement financier, les mentors supervisent entre un et quatre groupes. Certains établissements ont engagé une organisation communautaire pour faciliter le recrutement et l'encadrement des mentors ainsi que les rencontres des filles.

Pour plus de détails, s'adresser à Karen Austrian (kaustrian@ popcouncil.org) ou voir la page Web du projet sur http://www. popcouncil.org/projects/48_ SafeSmartSavingsVulnerableGirls.asp [en anglais].

Abriendo Oportunidades : Programme destiné aux filles indigènes de 8 à 18 ans des communautés rurales les plus pauvres du Guatemala Le programme Abriendo Oportunidades du Population Council est conçu pour atteindre et autonomiser les filles indigènes de 8 à 18 ans des communautés rurales les plus démunies du Guatemala. Le programme opère sur deux cohortes d'âge : de 8 à 12 ans et de 13 à 18 ans. Un modèle de leadership en cascade est utilisé au niveau de la communauté, où deux jeunes femmes de 15 à 18 ans issues de chacune dirigent chaque cycle annuel de jeunes participantes au club. Dans certaines communautés, une couche supplémentaire de leadership est introduite par l'implication de jeunes stagiaires féminines indigènes des ONG locales. Les filles leaders reçoivent un petit salaire en rémunération de leur animation des activités du club et présentent un autre modèle de rôle positif au niveau de la communauté ${ }^{17}$.

Pour plus d'information, s'adresser à Angel del Valle (adelvalle@popcouncil. org) ou voir la page Web du projet sur http://www.popcouncil.org/projects/ 244_CreateOpportunitiesMayan.asp.
Biruh Tesfa (Avenir prometteur) : Programme destiné aux employées de maison et migrantes rurourbaines dans les bas-quartiers d'Éthiopie

En Éthiopie, les adolescentes pauvres migrent souvent vers les centres urbains à la recherche de débouchés d'emploi et d'éducation ou pour échapper à la menace du mariage précoce. Certaines ont perdu un parent ou les deux au sida. Souvent non scolarisées, elles sont socialement isolées, sans protection et loin de leur famille. Ce qu'elles trouvent est un emploi de maison, où elles sont mal rémunérées et parfois battues ou violentées; certaines sont contraintes à se prostituer. Elles n'ont guère d'amies, personne à qui demander de l'aide, guère d'occasions de scolarisation et aucun lieu sûr où rencontrer d'autres filles et construire leurs réseaux de soutien. En partenariat avec la Commission pour les jeunes et les sports d'Addis Abeba et le ministère éthiopien de la Jeunesse et des Sports, le Population Council a conçu et mis en œuvre le programme " Biruh Tesfa " à l'intention des filles déscolarisées résidentes des bidonvilles. Le programme mobilise les filles en groupes de rencontre sous la conduite de mentors féminines adultes dans des espaces de la communauté. Après leur formation, les mentors identifient les filles admises à participer en se rendant de porte à porte dans la zone qui leur est affectée. Quand elles repèrent une candidate potentielle dans un ménage, elles l'invitent à participer au programme. Pour les filles intéressées, les mentors discutent la participation avec leurs parents, tuteurs ou - dans le cas des employées de maison leurs employeurs, dont la première réaction n'est souvent pas favorable. Les mentors sont responsables du suivi des filles qu'elles inscrivent au programme. Les groupes se rencontrent dans les salles et espaces communautaires que leur offre l'administration/le qebelé local.

Les filles reçoivent une formation couvrant notamment alphabétisation et calcul, communication et estime de soi, hygiène et menstruation, handicaps, VIH et sida, santé génésique, compétences financières et épargne, et violence de genre. Le projet remet à toutes ses participantes une carte d'identité revêtue de leur photo. Pour de nombreuses filles, cette simple carte est leur seule pièce d'identité et, souvent, la seule photo qu'elles ont d'elles-mêmes. Elles reçoivent aussi des cahiers, des stylos et des crayons. En raison de leur extrême pauvreté, beaucoup des bénéficiaires du programme n'ont pas accès à de simples articles de base tels que savon ou serviettes hygiéniques. Elles reçoivent chacune environ quatre pains de savon par an, ainsi que des serviettes hygiéniques réutilisables produites localement. Au moyen d'un système de bons, les filles de Biruh Tesfa peuvent bénéficier de services médicaux subventionnés ou gratuits auprès d'un réseau de prestataires. Une nouvelle initiative vise par ailleurs à accroître la participation des filles handicapées. En avril 2012, plus de 55 mille filles avaient bénéficié de l'intervention Biruh Tesfa dans 18 villes d'Éthiopie.

Pour plus de détails, s'adresser à Annabel Erulkar ( aerulkar@popcouncil. org) ou voir la page Web du projet sur http://www.popcouncil.org/ projects/41_BiruhTesfaSafeSpaces.asp [en anglais].

\section{RESSOURCES : PUBLICATIONS ET OUTILS}

Austrian, K. et D. Ghati. 2010. Conception d'un programme centré sur les filles: Boîte à outils pour l'élaboration, le renforcement et l'expansion de programmes destinés aux adolescentes. (Voir le chapitre 5 sur le développement du leadership et le mentoring.) Nairobi: Population Council. http://www.popcouncil. org/publications/books/2011_ AdolGirlsToolkit_fr.asp.

Promoting Healthy, Safe, and Productive Transitions to Adulthood. Série de bulletins d'information. New York : Population Council. http:// www.popcouncil.org/publications/ serialsbriefs/TABriefs.asp. 
- $\mathrm{N}^{\circ} 5$ : Equipping Mayan girls to improve their lives [en anglais]

- $\mathrm{N}^{\circ} 6$ : Addis Birhan project: Working with boys and men to address young girls' social vulnerability [en anglais]

- $N^{\circ} 12$ : Scaling up asset-building programs for marginalized adolescent girls in socially conservative settings: The Ishraq program in rural Upper Egypt [en anglais]

- $N^{\circ} 15$ : Tap and Reposition Youth (TRY): Providing social support, savings, and microcredit opportunities for young women in areas with high HIV prevalence [en anglais]

- $N^{\circ} 21$ : Biruh Tesfa provides domestic workers, orphans, and migrants in urban Ethiopia with social support, HIV education, and skills [accessible en français sous le titre "Biruh Tesfa procure aux employées domestiques, aux orphelines et aux immigrantes des zones urbaines éthiopiennes une assistance sociale, une formation sur le VIH ainsi qu'un programme de développement des compétences]

- $\mathrm{N}^{\circ} 30$ : Identifying sources of adolescent exclusion due to violence: Participatory mapping in South Africa [en anglais]

\section{NOTES}

1 Les enquêtes National Surveys of Adolescence réalisées par le Guttmacher Institute et ses partenaires au Burkina Faso, au Ghana, au Malawi et en Ouganda ont produit des données sur la densité des réseaux d'amitié, indiquant dans tous les groupes (ruraux/urbains, niveau de scolarité atteint) que les garçons ont en moyenne plus d'amis proches que les filles. (Biddlecom, Ann. 2008. Correspondance avec le responsable de la recherche) ; Hallman, K. 2005. "Gendered socioeconomic conditions and HIV risk behaviours among young people in South Africa ". African Journal of AIDS Research 4(1): 37-50.

2 Erulkar, A. et J. Matheka. 2007. Adolescence in the Kibera Slums of Nairobi Kenya. Population Council.
3 Erulkar, A. et al. 2004. Adolescent Life in Low Income \& Slum Areas of Addis Ababa, Ethiopia. Population Council.

4 Erulkar, A. et al. 2004. The Experience of Adolescence in Rural Amhara Region, Ethiopia. Population Council.

5 Lloyd, Cynthia B. 2009. New Lessons. New York: Population Council.

6 Salem, Rania, Barbara Ibrahim et Martha Brady. 2003. "Negotiating leadership roles: Young Women's Experiences in rural Upper Egypt ", Women's Studies Quarterly 31(3/4): 174-191.

7 Hallman, Kelly. 2008. "Social exclusion: The gendering of HIV risk in KwaZulu-Natal, South Africa. Document de travail. New York : UNESCO et Social Science Research Council.

8 Bruce, Judith et Kelly Hallman. 2008. "Reaching the girls left behind", Gender \& Development 16(2): 227 245.

9 Austrian, Karen, C. Ngurukie et C. Sakwa. 2009. "Integrating Financial Education and Savings Opportunities into Health Interventions for Adolescent Girls in the Kibera Slum of Nairobi, Kenya. " Document présenté à I'International Conference on Urban Health: Meeting Urban Health Needs Through Innovative Research, Policies, and Interventions. Nairobi, Kenya.

10 Sewall-Menon, Jessica, Judith Bruce et al. 2012. "The cost of reaching the most disadvantaged girls: Programmatic evidence from Egypt, Ethiopia, Guatemala, Kenya, South Africa, and Uganda. "New York: Population Council.

11 Par exemple, lors de la conception du programme Safe and Smart Savings Products (voir plus bas), une étude de marché a été réalisée sur plus de 200 filles de segments distincts (aînées, cadettes, scolarisées, déscolarisées). L'étude a recueilli les opinions relatives aux caractéristiques des comptes d'épargne, mais aussi aux services non financiers, aux sujets de formation et à la logistique du programme.

12 Austrian, Karen et Dennitah Ghati. 2010. Conception d'un programme centré sur les filles : Boîte à outils pour l'élaboration, le renforcement et l'expansion de programmes destinés aux adolescentes. Nairobi :
Population Council. (Outil d'évaluation conceptualisé par Judith Bruce et Karen Austrian.)

13 Bruce, Judith. 2012. "La violence faite aux adolescentes : Un défi fondamental à la vraie égalité ", une publication Girls First! New York : Population Council.

14 Par exemple, à Binti Pamoja, les mentors se réunissent une fois par mois avec les agents de terrain pour partager leurs succès et leurs difficultés ; chaque trimestre, elles révisent leurs plans de travail en fonction des besoins de leurs groupes. Dans le programme Safe and Smart Savings Products, des formations de recyclage mensuelles d'une journée sont offertes aux mentors sur les lacunes qu'elles identifient ellesmêmes dans leurs connaissances ou compétences.

15 Horizons/Population Council. 1999.

" Peer Education and HIV/AIDS:

Past Experience, Future Directions. " Washington, DC, et New York: Population Council.

16 Adamchak, Susan. "Youth Peer Education in Reproductive Health and HIV/AIDS: Progress, Process, and Programming for the Future. Youth Issues Paper No. 7. "Arlington, VA: Family Health International/YouthNet Program.

17 Catino, Jennifer. 2012. "La santé des adolescentes vulnérables : un investissement stratégique doublement rentable ", une publication Girls First! New York: Population Council. 



\section{(2) Population Council}

Le Population Council s'attelle aux questions cruciales de la santé et du développement - qu'il s'agisse d'endiguer la progression du VIH, d'améliorer la santé reproductive ou d'offrir aux jeunes la promesse d'une vie satisfaisante et productive. Par ses travaux de recherche en biomédecine, en sciences sociales et en santé publique dans 50 pays, il s'efforce d'apporter avec ses partenaires des solutions qui conduisent à des politiques, des programmes et des technologies plus efficaces, pour améliorer la vie partout dans le monde. Fondé en 1952, le Population Council est une organisation non gouvernementale à but non lucratif. II siège à New York sous la conduite d'un conseil d'administration international. 